\title{
Possibilidades e limites na criação coletiva de artigos da Wikipédia: o artigo "Vitor Marinho de Oliveira"
}

Leon Ramyssés Vieira Dias, UFRJ, leondias@ufrj.br

André Malina, UFRJ, andremalina@yahoo.com.br

Silvio Telles, UFRJ/UERJ, silviotelles@ufrj.br

Bianca Miarka, UFRJ, miarkasport@hotmail.com

Ângela Celeste Barreto de Azevedo, UFRJ, angelaestagio@yahoo.com.br

Resumo: No presente estudo, discutiu-se, a partir da análise da criação do artigo "Vitor Marinho de Oliveira", - um intelectual vinculado à área sociocultural da Educação Física - o fenômeno da Wikipédia, uma enciclopédia organizada em hipertexto e de colaboração cooperativa. Para tal discussão, descreveu-se o processo de criação e submissão do artigo na Wikipédia, apontando os limites e as possibilidades, bem como a interação com a própria plataforma. Utilizou-se como referencial teórico as concepções de Álvaro Vieira Pinto sobre a tecnologia, além de autores que trataram especificamente da Wikipédia. Como principais resultados, foram encontradas limitações para publicação de artigos na Wikipédia, embora os critérios de criação coletiva do conhecimento, tratando-o como um bem público e com difusão gratuita, atenda a uma perspectiva crítica da tecnologia. Para além disso, recomenda-se aos pesquisadores da Educação Física a massificação de publicações no ciberespaço visando preencher essa lacuna e dar usufruto aos leitores da Wikipédia sobre questões relevantes da área, incluindo o conhecimento dos seus principais intelectuais.

Palavras chave: hipermídia; conhecimento; tecnologia; internet; Educação Física.

\section{Possibilities and limits on the collective creation of Wikipedia articles: the article "Vitor Marinho de Oliveira"}

Abstract: In the present study, it was discussed, from the analysis of the creation of the article "Vitor Marinho de Oliveira", - an intellectual linked to the socio-cultural area of Physical Education - the Wikipedia phenomenon, an encyclopedia organized in hypertext and with cooperative collaboration, was discussed. For this discussion, the process of creating and submitting the article on Wikipedia was described, pointing out the limits and possibilities, as well as the interaction with the platform itself. Álvaro Vieira Pinto's conceptions about technology were used as a theoretical framework, in addition to authors who dealt specifically with Wikipedia. As main results, limitations were found for publication of articles on Wikipedia, although the criteria of collective knowledge creation, treating it as a public good and with free diffusion, is in accordance with a critical technology perspective. In addition, it is recommended that Physical Education researchers massify publications in cyberspace in order to fill this gap and give Wikipedia readers access to relevant issues in the area, including the knowledge of their main intellectuals.

Keywords: hypermedia; knowledge; technology; internet; Physical Education.

\section{INTRODUÇÃO/CONTEXTUALIZAÇÃO}

A Educação Física (EF) abarca conteúdos de diferentes áreas do conhecimento, e é fundamentada em diversas abordagens relacionadas à concepção, ensino e relevância 
social. Os fenômenos abordados na $\mathrm{EF}$ debatidos nos canais midiáticos são vinculados à saúde, ao esporte e ao lazer. Esses conteúdos estão disseminados socialmente, sendo comunicados e discutidos - independentemente do valor acadêmico-científico -, dentre outros, via mídia.

Já os intelectuais que fundaram e trataram academicamente o conhecimento intrínseco da $\mathrm{EF}$, normalmente não são lembrados ou apresentados ao grande público, mesmo tendo relevante influência na área. Ocorre diferentemente quando se trata, por exemplo, de intelectuais oriundos da EF que são militantes do esporte ou expoentes esportivos, e mesmo de intelectuais de outras áreas.

Nesse sentido, atribui-se às diferentes mídias o papel de difusoras de informações para o grande público, devido à possibilidade e capacidade de alcance. Partindo desse pressuposto, a internet pode induzir a construção e difusão de ideias oriundas da EF e de intelectuais relevantes no seu desenvolvimento. Pode-se considerar, assim, que a biografia desses sujeitos ao retratar aspectos da vida profissional e acadêmica faz parte do próprio conteúdo intrínseco da EF.

Partindo-se do entendimento que pode haver falta de difusão do conhecimento proveniente de ideias e de intelectuais oriundos da EF e que, por outro lado, os conteúdos da área associados à mídia são difundidos, mas sem vinculação explicitada com a EF, cabe apresentar o seguinte problema de investigação: Quais impasses e soluções apresentadas para a difusão dos intelectuais vinculados à EF por meio dos ciberespaços?

Como objeto de análise foi escolhido a Wikipédia, um projeto de enciclopédia virtual e livre de licença. A Wikipédia está disponível para acesso em vários países e em diferentes idiomas, na qual os próprios usuários são colaboradores, possuindo mais de 43 milhões de artigos (Wikipédia, 2018). Nesses termos, o objetivo da presente pesquisa é verificar se o escopo teórico da Wikipédia se aproxima ou distancia-se de uma perspectiva crítica da tecnologia e, especificamente apontar o papel que a tecnologia assume na difusão do conhecimento por via dos ciberespaços, especificamente das enciclopédias virtuais. Para tanto, optou-se por realizar uma análise da construção de artigos na Wikipédia e dos próprios verbetes ligados aos intelectuais da EF.

O referencial que embasa o corpus teórico desse artigo é o de Álvaro Vieira Pinto (2005). Este autor compreende e formula suas considerações sobre o conceito de tecnologia na perspectiva do pensamento crítico. Para ele, há uma ciência da técnica enquanto fato concreto que carece de indagação epistemológica. Logo, considera a tecnologia como epistemologia da técnica e que a tecnologia proporciona edificar ponderações sobre a técnica, sugerida por reflexões críticas e permitindo o alcance do nível da teorização.

A seguir, no item 2, será descrito os passos metodológicos adotados na pesquisa. Já no item 3, será feita uma breve descrição da Wikipédia e de seus aspectos, bem como uma análise das possibilidades e dos limites na criação de um artigo na plataforma, a partir da criação do verbete "Vitor Marinho de Oliveira". Por fim, no item 4, serão realizadas as considerações pertinentes ao estudo.

\section{PASSOS METODOLÓGICOS}

Inicialmente, foi feita uma seleção de intelectuais da EF brasileira que produziram conhecimentos considerados importantes para o desenvolvimento da área. Listamos assim cinquenta (50) intelectuais, distribuídos por referências de época, especificamente anos de 1980, 1990 e 2000. Em seguida, verificou-se quantos tinham 
suas biografias publicadas na Wikipédia. Foram encontrados quatro artigos: "João Paulo Medina", "Vitor Marinho de Oliveira", "Elenor Kunz" e "Manoel Tubino".

Para chegar a tal seleção consultou-se algumas produções que tratam sobre os intelectuais da EF e a importância deles para as transformações que ocorreram na área. Destas, destacam-se Dias (2018), Malina (2017; 2005) e Daólio (1997). Desse modo, a listagem inicial dos intelectuais pesquisados partiu dessas (e de outras) produções, sem desconsiderar, no entanto, outros intelectuais que também possam ser importantes, visto a difusão de seu trabalho e o alcance de suas produções. Ainda assim, não se ampliou tal listagem. Desse modo, a opção metodológica utilizada dispensou enquetes ou questionários de seleção, perfazendo uma amostragem de escolha notória.

Tratando-se especificamente de intelectuais brasileiros da EF que produziram conhecimento na área, João Paulo Subirá Medina e Vitor Marinho de Oliveira foram responsáveis, dentro outros, pelo movimento chamado de pensamento renovador e consequente perspectiva sociocultural na década de 1980, no qual efervesceram ideias e intelectuais da EF (Malina; Azevedo, 2017). Elenor Kunz, também fruto desses anos, lançou, em 1994 na forma de livro, as bases da abordagem crítico-emancipatória. Esta abordagem seria, posteriormente, amplamente utilizada e aprofundada na EF brasileira. Já Manoel José Gomes Tubino era militar de formação, atuou, inicialmente, ainda nos anos 1970 com Treinamento Desportivo e, posteriormente, destacou-se, como expoente na política ligado ao desenvolvimento do esporte e, por conseguinte, à Educação Física. Coordenou importantes instituições da EF como a Fédération Internationale $d$ Education Physique (FIEP).

Tendo João Paulo Subirá Medina, Vitor Marinho de Oliveira e Elenor Kunz como referências da perspectiva sociocultural da EF, e de Manoel José Gomes Tubino como um intelectual que transitou da área da biodinâmica à sociocultural, compreendese que suas próprias trajetórias profissionais e acadêmicas, materializadas, dentre outros, em livros, poderia ser suficiente para gerar conteúdo digital relevante. Como pressuposto, pode-se afirmar que os anos de 1980 e seus desdobramentos foram um momento histórico importante de ser publicizado, já que modificou os rumos da área, embora pareça ter sido veiculado e recebido com interesse mais restrito ao meio acadêmico-científico.

Dos três artigos encontrados, no entanto, foram excluídos da análise: a) o artigo "João Paulo Medina", pelo fato de não retratar trajetória e importância de João Paulo Subirá Medina na EF, especificamente. A sua biografia está restrita às realizações enquanto destaque no meio futebolístico. Esse realce pode ser visualizado, por exemplo, quando afirma sua passagem por diversos clubes de futebol. Em que pese a sua importância para o desenvolvimento desse esporte, que é um subconteúdo da EF, não há referência a sua importante atuação na construção e desenvolvimento intrínseco à área; b) o artigo "Elenor Kunz", pois nas seis linhas que compõem o artigo somente é abordada a formação acadêmica do autor e um de seus livros na área da EF. Ademais se entende que, por ser um artigo muito curto, não houve empiria para apontar e analisar limites e possibilidades em sua construção; c) o artigo "Manoel Tubino" também está muito curto, não sendo mencionadas diversas informações de sua extensa trajetória de atuação desde os anos de 1960.

Desse modo, por não possuir os critérios de exclusão dos artigos "João Paulo Medina", "Elenor Kunz" e "Manoel Tubino", o artigo "Vitor Marinho de Oliveira" foi escolhido para análise. 
A partir do artigo "Vitor Marinho de Oliveira" procurou-se evidenciar as questões inerentes ao processo de criação da página. Para tanto, foram coletados dados referentes às edições e publicações do artigo, no link "ver histórico", que retratam seu processo de criação. Nesse mesmo local (link "ver histórico"), também foi possível visualizar mensagens entre colaboradores/usuários e administradores. Assim, observouse um total de cinquenta e cinco (55) notificações/alterações na página desde sua criação, sendo quarenta e quatro delas referentes à criação e adequação do artigo "Vitor Marinho de Oliveira" à Wikipédia. Essas quarenta e quatro (44) notificação/alterações foram analisadas visando compreender as possibilidades e as limitações de se publicar na enciclopédia virtual e livre da Wikipédia.

Nesses termos, o estudo foi divido em duas partes. Na primeira parte (item 3.1), verificou-se a possibilidade de construção coletiva dos artigos da Wikipédia, a partir do levantamento das informações presentes no site da Wikipédia. Desse modo, utilizaramse os conteúdos referentes à criação da Wikipédia, seus objetivos e políticas.

$\mathrm{Na}$ segunda parte (item 3.2), em posse do histórico de edição do artigo "Vitor Marinho de Oliveira" e amalgamado pela base teórica de Vieira Pinto (2005), foi analisado como ocorre à criação de um artigo na Wikipédia. Para subsidiar tal análise, quando necessário, recorreu-se aos critérios que norteiam os usuários na criação e edição de artigos, como: 1) os Cinco Pilares; 2) os Critérios de Notoriedade; 3) a Lista de Políticas; 4) o Tutorial; 5) o Guia de Edição/Formatação e; 6) a página de Discussão do Usuário.

\section{DESCRIÇÃO, ANÁLISE E DISCUSSÃO DOS DADOS}

\subsection{Sobre a Wikipédia}

O conhecimento produzido na área da EF é difundido tanto por meio acadêmicocientífico quanto por fontes alternativas. Cabe compreender, no entanto, que o conhecimento desenvolvido fora do espaço acadêmico pode ser apropriado para estudo pelas universidades e, posteriormente, difundido pelos seus próprios meios de formação e informação. A relação contrária também pode acontecer e o conhecimento produzido nas universidades pode ser difundido por meio dos ciberespaços.

Os modos de transmissão comunicativa via mídia estão submetidos aos arranjos diversos produzidos pelo formato de transmissão. Por isso, um jogo de futebol que se assiste na televisão, por exemplo, deverá guardar características específicas do veículo de comunicação (Silveira; Silva, 2016). Esses veículos de informação e formação vêm ganhando maior visibilidade por conta, principalmente, do advento da internet. Monteiro (2017) atenta que a criação da internet possibilitou, além de novas plataformas midiáticas esportivas, a geração de espaços de organização do conhecimento e de fácil acesso, como é o caso das enciclopédias virtuais, que concorrem e ganham espaço no campo da informação, utilizadas de forma pragmática na busca dos mais diferentes conteúdos.

Entender o papel dessas enciclopédias virtuais nos ciberespaços para formação e informação dos sujeitos compreende, necessariamente, reconhecer o contexto histórico em que essas ferramentas surgiram e se propagam. Nesse sentido, o surgimento de novos segmentos da mídia produziu espaços virtuais de "produção de saberes".

Pesquisadores tem se voltado para as enciclopédias virtuais com estudos sobre diversos temas. Muitos desses abordam especificamente a Wikipédia, enciclopédia livre de licença, na qual os usuários são colaboradores, como é do caso dos artigos de 
Monteiro (2017), Ferreira (2013), Burke (2012), Ribeiro e Gottschalg-Duque (2011), Vieira e Christofoletti (2008), Bottentuit Junior e Coutinho (2008), Johnson (2008), Lima (2006), Moreira (2005).

A Wikipédia "é derivada da Nupédia, uma Enciclopédia online paga e de edição restrita" (Ribeiro; Gottschalg-Duque, 2011, p. 173). Foi lançada em 15 de janeiro de 2001 por Jimmy Wales e Larry Sanger e atualmente é mantida pela Wikimedia (Wikipédia, 2018). A Wikimedia como proprietária dos direitos da Wikipédia descreve em sua página oficial que o propósito atual do grupo, aprovado pela Resolução de Conselho em abril de 2007, é de

empoderar e engajar pessoas pelo mundo para coletar e desenvolver conteúdo educacional sob uma licença livre ou no domínio público, e para disseminá-lo efetivamente e globalmente[...] A Fundação irá disponibilizar e manter disponíveis as informações úteis de seus projetos gratuitamente através da Internet, para sempre (WIKIMÉDIA, 2017, s/p).

A reconhecida popularidade por parte de pesquisadores traz várias indagações quanto à veracidade das informações difundidas na rede. Para Primo e Recuero (2003) a Wikipédia trata de hipertextos cooperativos, assim como os blogs, possibilitando a construção, a organização e a difusão do conhecimento pelos próprios internautas, caracterizando uma "web viva". Já na visão de Canin (2013), os canais de comunicação podem configurar-se como colaboradores de formação do senso comum na esfera pública, instaurando consensos públicos pelo mercado cultural.

Por outro lado, o fato de ser colaborativa pode, na visão de Ribeiro e Golttschalg-Duque (2011), levar a distorções em artigos, atrapalhando a formação de critérios de confiabilidade. Rodrigues (2010) atenta ainda para a construção do conhecimento na Wikipédia, alimentada por colaboradores que compartilham de diferentes ideias, ideologias e níveis de informação.

Diferentemente de enciclopédias convencionais, os artigos encontrados na Wikipédia foram escritos de forma conjunta por voluntários de diferentes países, podendo ser editados por qualquer pessoa com acesso à internet e com uma conta no sítio da Wikipédia, podendo haver um conteúdo informacional acadêmico e nãoacadêmico.

Cabe, portanto, verificar o processo de construção coletiva em uma experiência prática visando observar como tal processo de difusão do conhecimento está posto na Wikipédia diante de uma organização social do tipo capitalista, com uma racionalidade, por vezes, a-histórica e cientificista. Para tanto, segue à frente a criação de um artigo para publicação nessa enciclopédia virtual.

\subsection{Vitor Marinho de Oliveira: análise das possibilidades e limites na criação de um artigo/verbete na Wikipédia}

Com a finalidade de compreender limites e possibilidades de uso da Wikipédia, observou-se como ocorreu a criação do artigo "Vitor Marinho de Oliveira", que aborda parte de sua trajetória profissional, pessoal e acadêmica, sua relevância para a EF no cenário nacional e explanações sobre a sua obra. Ao perscrutar-se o histórico de edições da página foi possível verificar o processo de construção e publicação do artigo, encontrando-se duas dificuldades nesse processo: (a) adequar o texto às políticas de publicação da Wikipédia, bem como a dificuldade de compreensão do colaborador/usuário aos tópicos relacionados à política de publicação; (b) tornar aceita a 
relevância social do biografado da área de EF aos administrados responsáveis pela Wikipédia.

A dificuldade em adequar um texto às políticas da publicação do Wikipédia pode ser retratada nas mensagens ficaram estampadas na página do artigo, como:

\footnotetext{
1- "Este artigo não está em nenhuma categoria. Por favor, categorize-o para que seja listado com suas páginas similares";

2- "Esta página ou secção cita fontes confiáveis e independentes, mas que não cobrem todo o conteúdo. Por favor, adicione mais referências e insira-as corretamente no texto ou no rodapé".

3- "Material sem fontes poderá ser removido. Encontre fontes: Google (notícias, livros e acadêmico)".

4- "Esta página ou seção precisa ser wikificada. Por favor ajude a formatar esta página de acordo com as diretrizes estabelecidas".

5- "Esta página precisa ser reciclada de acordo com o livro de estilo. Sinta-se livre para editá-la para que esta possa atingir um nível de qualidade superior".
}

Cabe ressaltar que as orientações para a criação de verbetes estão disponíveis no próprio site da Wikipédia (Wikipédia, 2018) e caso algo não atenda ao escopo da plataforma, o colaborador é notificado por mensagens automáticas (como as destacadas acima) ou por outro colaborador que assume a função de administrador.

A questão principal que pode ser observada nas mensagens é referente à divulgação de conteúdos inéditos, provenientes de uma pesquisa acadêmico-científica e que seguem um rigor metodológico comum no âmbito da pesquisa no ensino superior. Tais pesquisas podem revelar questões ainda não discutidas, sendo, portanto, um conteúdo inédito. Segundo a própria Wikipédia (2018):

[...] a Wikipédia deve expor o conhecimento que já está estabelecido e reconhecido - em outras palavras, não deve apresentar, por exemplo, novas informações ou obras originais. Uma alegação que é suscetível de ser questionada requer pelo menos uma referência a uma fonte de confiança. Entre os editores da Wikipédia, isto é frequentemente descrito como "comprovação, não a verdade", para expressar a ideia de que os leitores, e não a enciclopédia são responsáveis por verificar a veracidade dos artigos e fazer as suas próprias interpretações. Finalmente, a Wikipédia não deve tomar partido. Todas as opiniões e pontos de vista, se imputáveis a fontes externas, deverão ser adequadamente cobertos pelo texto do artigo. Isto é conhecido como ponto de vista neutro $[\ldots]$.

Vitor Marinho foi um dos criadores e responsável pela ascensão do pensamento renovador e perspectiva sociocultural da EF na década de 1980. Este intelectual escreveu, dentre outros, os livros "O que é Educação Física", de 1983, e "Educação Física Humanista", de 1985, "Consenso e Conflito da Educação Física Brasileira" de 1994 e "O Esporte Pode Tudo" de 2010. Em sua trajetória pela EF, trouxe para a área o debate sobre questões ideológicas, fez críticas ao tecnicismo e difundiu a pedagogia humanista em contraposição ao comportamentalismo.

Ainda sobre as dificuldades em adequar o texto acadêmico às políticas da publicação do Wikipédia e a dificuldade de compreensão de alguns tópicos relacionados à política de publicação, as mensagens que ficaram estampadas na página do artigo "Vitor Marinho de Oliveira" podem ser compreendidas, na visão de Monteiro (2017), como "autocríticas", pois avisam sobre os riscos à 'saúde' intelectual do usuário. Isso 
porque o conhecimento enciclopédico refletiria definições pragmáticas de índices contemporâneos. Ainda para Monteiro (2017), a enciclopédia tem desenvolvido algoritmos e agentes inteligentes para agregar semântica à Wikipédia e à indexação e busca de conteúdo no ciberespaço.

Notou-se que a criação do artigo "Vitor Marinho de Oliveira" buscou seguir os preceitos dos Cinco Pilares, bem como as recomendações presentes nos Critérios de Notoriedade, na Lista de Políticas, no Tutorial e no Guia de Edição/Formatação. Também se observou que, imediatamente após sua criação, o artigo tornou-se público sendo possível a qualquer um visualizá-lo. Foi necessário, no entanto, legitimar a relevância social do biografado para que o artigo fosse mantido.

Os Critérios de Notoriedade da Wikipédia são responsáveis por estabelecer se alguns artigos serão enviados para discussões de eliminação de artigos. Os critérios presentes na plataforma da Wikipédia (2018) apontam que "um tópico é presumido como notável se recebeu cobertura significativa de fontes reputadas e independentes do assunto tratado". Logo, são necessárias fontes que cubram todo o texto sobre determinado assunto, independente do canal de comunicação, para que a publicação, segundo a plataforma, seja considerada um artigo e não um esboço.

Para que o artigo tenha relevância - quando é o caso de uma biografia, conforme visto na criação do artigo "Vitor Marinho de Oliveira" -, torna-se necessário: a) demonstrar a relevância social do biografado; b) passar por uma análise de um administrador sobre a relevância de um artigo sendo que, não necessariamente esse administrador tem domínio sobre o assunto do biografado.

A notoriedade de Vitor Marinho justifica-se na própria história da EF. Na década de 1980, junto a outros intelectuais, Vitor Marinho trouxe para a área o debate sobre as questões ideológicas. Nesse período, aprofundaram-se os debates sobre a EF, culminando em correntes ou tendências que contestavam o caráter biológico da área, além das influências militar e os aspectos da pedagogia tradicional presente no âmbito escolar. O autor ressaltou a importância da EF para as questões relacionadas à saúde e ao esporte, porém, afirmando que as questões de caráter político-ideológico quando negligenciadas pelos professores reforçavam a perspectiva tecnicista da prática docente.

Vitor Marinho atuou com pesquisas na área da Educação com o objetivo de estudar e explicar os fenômenos da própria EF. Produziu livros e artigos que ganharam notoriedade no país inteiro, abordando temas como: História da EF, Sociologia da EF, EF Escolar etc.

Com Vieira Pinto (2005), pode-se compreender que a negação dos determinantes históricos para o entendimento das questões deste tempo histórico caracteriza uma visão ingênua e acrítica. Desse modo, pode-se compreender que a negação da relevância de Vitor Marinho e, consequentemente, a negação do movimento renovador da EF, é negar os determinantes históricos necessários para entender a EF nos dias atuais.

Logo, na visão de Vieira Pinto (2005) os meios que compartilham de tal perspectiva podem estar associados tanto à ideia de tecnologia como de técnica, observado nos discursos daqueles que não se apropriam de um embasamento teórico apropriado. Esta seria, na visão do autor, uma definição coloquial e de senso comum. Estabelecendo as devidas mediações com essa concepção de tecnologia criticada por Vieira Pinto (2005), o conhecimento que descarta as múltiplas determinações históricas de determinado fato, pode se distanciar de uma perspectiva crítica, recaindo no senso comum.

A criação do artigo que traz a biografia intelectual de Vitor Marinho mostrou intensa dificuldade de aceitação pelo administrador da Wikipédia, haja vista que tem a 
função de verificar se as normas da plataforma estão sendo cumpridas por seus colaboradores, bem como verificar a relevância e a fidedignidade das informações prestadas pelos usuários. Segundo a Wikipédia (2018), estão atribuídas ao administrador as competências de: proteger, desproteger e editar páginas protegidas; eliminar e restaurar páginas e revisões específicas; visualizar páginas e imagens eliminadas e os seus respectivos históricos; reverter à última edição de um artigo; ocultar vandalismos na página de mudanças recentes, bem como outras funções relacionadas à segurança dos usuários e das informações.

O administrador da Wikipédia, para ser qualificado como tal, deve ser um usuário com registro de no mínimo seis meses na plataforma e deve ter realizado duas mil edições válidas no domínio principal. Além disso, é necessário que o usuário se candidate à função devendo passar por um processo de votação, de acordo com a política de administradores (Wikipédia, 2018). O critério de seleção do administrador não está relacionado ao conhecimento específico da área e, desse modo, torna-o uma espécie de "parecerista" não qualificado para determinados assuntos. Devido à diversidade de conteúdos presentes na Wikipédia, os administradores podem julgar como relevante o que é previamente difundido nos meios de comunicação de massa. Ratifica-se assim, a concordância com Trevisol e Novello (2014), de que na sociedade pós-moderna a visibilidade é uma das principais características proporcionadas de forma instantânea e global.

Passar pela análise de um administrador sobre a relevância de um artigo sendo que, não necessariamente esse administrador tem domínio sobre a importância do biografado, pode ser compreendido à luz de Vieira Pinto (2005), como o trabalho dos pensadores ingênuos, aqueles que pensam a tecnologia pelo seu produto, desconsiderando os aspectos filosóficos. Para o autor, estes agem verdadeiramente como técnicos, e entendem a tecnologia como o modo de produzir alguma coisa, ou seja, a teoria, a ciência e o estudo de determinada finalidade, sendo nesse caso, uma instância maior a qual toda coisa a ser produzida está submetida. É a ideologização da técnica.

Nessa perspectiva, junto às formulações dos chamados pensadores que têm uma visão determinista, existencialista e essencialista sobre a tecnologia, os conceitos passam a ser estabelecidos e se tornam consenso, de forma a se reproduzirem em todo meio social. Ao contrário, o desenvolvimento tecnológico é resultado de uma acumulação histórica de conhecimento e da prática social. A exaltação feita ao desenvolvimento sistemático de novas tecnologias mostra carência e atraso na medida em que há uma teleologia entre tecnologia do presente e tecnologia do futuro (Vieira Pinto, 2005).

O grau de exigência proposto pela Wikipédia mostrava um somatório de questões aliando a transformação da linguagem técnica para uma decodificação dos usuários carregada de preconceitos com a área de EF, mostrando uma ambiguidade entre socialização do conhecimento e desconfiança da relevância daquele conhecimento. Além disso, se o conhecimento é histórico, as circunstâncias históricas do mundo atual estão presentes na geração de conhecimentos da Wikipédia.

Oposto às concepções acríticas de tecnologia, no entanto, o entendimento da tecnologia à luz de uma consciência crítica permite aos técnicos e a todos que se apropriam da tecnologia refletirem sobre os aspectos do trabalho profissional de modo a alcançar uma imagem teórica de sua realidade existencial. Sem esse aporte teórico sobre 
as técnicas, o homem fica desprovido de boas respostas quando indagados sobre o que fazem e porquê fazem (Vieira Pinto, 2005).

Atualmente, há grande proliferação tecnológica. Utilizando-se da dialética podese compreender que o desenvolvimento tecnológico é resultado de uma acumulação histórica de conhecimento e da prática social (Vieira Pinto, 2005). O conteúdo presente no artigo "Vitor Marinho de Oliveira", criado na Wikipédia, obedece à concepção dialética do pensamento, pois ao mesmo tempo em que apresenta a vida e a obra de um sujeito histórico da EF brasileira, trás elementos do passado, mas que ajudam a pensá-la nos dias de hoje.

Apesar das dificuldades de legitimação da notoriedade de Vitor Marinho que foram observadas no processo de criação e difusão do artigo, a possibilidade de construção do conhecimento de forma colaborativa e coletiva pretendido pela Wikipédia traz a visão de que o conhecimento acumulado pela humanidade é de origem social. Nesse aspecto, os artigos gerados pela Wikipédia seriam desenvolvidos pelas pessoas de forma geral, para resolver necessidades colocadas pela sociedade. Desse modo, o conhecimento seria disponibilizado em escala e poderia ser acumulado em um processo cooperativo, portanto a partir de uma natureza social.

À luz de Vieira Pinto (2005), pode-se inferir que a Wikipédia possibilita a construção e a difusão de um conhecimento crítico. Nesse caso específico, porque o pensamento de Vitor Marinho ainda influencia professores e estudiosos da EF em todo o Brasil. As perspectivas apontadas por ele ajudam a pensar em uma EF crítica, humanista e transformadora a partir da problematização dos seus conteúdos.

Inspirado em Vieira Pinto (2005), pode-se aludir sobre a assimilação da consciência crítica pelos técnicos, ou seja, os que auxiliam na disseminação da tecnologia proveniente da Wikipédia, não possuem necessariamente conhecimento filosófico para tanto, relegando ao segundo plano a fundamentação e o conhecimento necessários para compreensão daquilo que lhes é ofertado e do que deve ou não ser difundido como conhecimento necessário e relevante. Nesse sentido, ratificam a manutenção do status quo vigente, no caso a manutenção e ampliação dos artigos de pessoas por características não investigadas.

Assim sendo, o processo de criação de artigos na Wikipédia pode ser observado por dois prismas com conotações negativas e positivas: 1- pode gerar questionamentos referentes à fidedignidade dos conteúdos, superficialidade das informações, banalização da pesquisa científica, reprodução do senso-comum, plágio e falta de qualidade da escrita; 2- A plataforma pode ser uma ferramenta pedagógica, não apenas informativa, mas também como provedor ao exercício da escrita, possibilitando aos estudantes criar artigos de forma clara e sucinta para públicos heterogêneos. No entanto, as conotações positivas no uso da Wikipédia podem minimizar as negativas, conforme o usuário tem a possibilidade de consultar as referências e ir à fonte primária daquele texto, averiguando sua veracidade e, se necessário, alterá-lo, seja na redação ou no conteúdo.

Desse modo, é necessário compreender que a seleção dos conteúdos, ainda mais quando se trata sobre a vida de um sujeito, obedece às bases do modo de produção dessa sociedade que pode ocasionar o desconhecimento da ação racional humana. Logo, os biografados têm a imagem vinculada a meios que atendem à demanda da sociedade capitalista, como os canais de rádio e televisão, além da própria internet. Pelos indicativos, a maneira adotada pela Wikipédia para selecionar os conteúdos, bem como verificar a fidedignidade das informações contidas nos artigos criados, não acompanha uma perspectiva crítica de tecnologia. Ao contrário, a tecnologia se manifestaria como 
ideologização da técnica e nesse sentido, visto que vivemos em uma sociedade de modo de produção capitalista, a tecnologia se apropriaria de todos os recursos possíveis para sustentar seu modelo.

De outra forma, a seleção de conteúdos nas enciclopédias virtuais manifesta, de forma implícita ou explícita, as questões de ordem político-ideológicas, principalmente quando a perspectiva de relevância do conteúdo analisado por um administrador está atrelada à influência da mídia. Assim, as ferramentas de informação que se difundem por meio da internet podem reiterar consensos hegemônicos da sociedade capitalista, distanciando-se de uma perspectiva crítica de tecnologia.

Assim, as relações estabelecidas na construção do artigo exposto na página do professor Vitor Marinho apontaram significativas dificuldades encontradas para materializá-lo diante das desiguais exigências impostas, conforme o biografado. Dessa forma, pode-se apontar nesse caso que a concepção de notoriedade da Wikipédia parece estar ligada à visibilidade midiática do biografado e não a sua importância intelectual ou factual. Nesses termos, evidenciaram-se limitações para publicar no ciberespaço da enciclopédia virtual Wikipédia.

\section{CONSIDERAÇÕES FINAIS}

O desenvolvimento tecnológico possibilitou uma maior difusão do conhecimento científico e não-científico. Com isso, também foram desenvolvidas novas formas de organização e veiculação desse conhecimento, principalmente por meio da internet. À medida, no entanto, em que surgem novas possibilidades de formação e informação também surge a possibilidade dessas ferramentas serem apropriadas pelo capitalismo na figura de um mercado de difusão cultural. Nessa perspectiva, o conhecimento produzido e disseminado no ciberespaço pode servir a interesses hegemônicos, resultado do processo de digitalização da informação. Em meio a essa questão, as enciclopédias virtuais refletem as tensões presentes na sociedade.

De maneira geral, entretanto, o escopo teórico da Wikipédia sugere como tese a apropriação e disseminação colaborativa e de acesso público ao conhecimento, sem ônus para os seus usuários. Tal tese coincide, na forma axiológica, com a visão do conhecimento como "um bem público" presente na perspectiva crítica de tecnologia.

Desse modo, como visto em Vieira Pinto (2005) pode-se ressaltar a necessidade de uma tecnologia que permita ao homem compreender sua realidade existencial, uma vez que "o domínio teórico da técnica pelo homem liberta-o da servidão prática à técnica, que vem sendo, crescentemente, o modo atual de vida pelo qual é definido e reconhecido" (p.223).

De ponto de vista específico, o processo de criação do artigo "Vitor Marinho de Oliveira" traz elementos sobre os critérios de aceitação e publicização de artigos na Wikipédia. A possibilidade de se criar ou editar um artigo a qualquer momento e tê-lo imediatamente disponível para visualização em qualquer lugar do mundo, demonstra umas das principais facilidades da Wikipédia. Isso também pode estar atrelado à escolha dessa ferramenta como forma de publicizar informações das mais diferentes esferas. A atração pelas facilidades desta enciclopédia virtual, no entanto, pode revelar dificuldades implícitas no processo de criação de artigos, como foi o caso do artigo Vitor Marinho de Oliveira.

Por fim, cabe recomendar aos pesquisadores vinculados à Educação Física que utilizem o espaço da Wikipédia publicizando questões relevantes para 0 desenvolvimento da área, inclusive a difusão de seus principais intelectuais. Dessa 
forma, pode-se tentar minimizar ou neutralizar dificuldades inerentes àquilo que é expressado pelos administradores da página e avaliadores dos conteúdos.

\section{REFERÊNCIAS}

BOTTENTUIT JUNIOR, J; COUTINHO, C. P. Wikis em Educação: potencialidades e contextos de utilização. In: Ana A. Carvalho (Org.), Actas do Encontro sobre Web 2.0 (pp. 336-341). Braga: CIED, 2008.

BURKE, P. Uma história social do conhecimento II: da Enciclopédia à Wikipedia.

Rio de Janeiro: Zahar, 2012.

CANIN, D.O. Mídia massiva e legetimidade: a notoriedade das ONGs e temas sociais pelas lentes dos folhetins na Rede Globo. In: Encontro Nacional de História da Mídia, Ouro Preto, 2013.

DAÓlIO, J. Educação Física Brasileira: Autores E Atores Da Década De 80. Campinas: s.n., 1997.

DIAS, L. R. V. Técnica e política na obra de Vitor Marinho: uma análise teóricoepistemológica. Rio de Janeiro, 2018. Dissertação (Mestrado profissional em Tecnologia para o Desenvolvimento Social) - Centro de Tecnologia, Universidade Federal do Rio de Janeiro, Rio de Janeiro, 2018.

FERREIRA, A. C. F. Discursos sobre Cidades na Enciclopédia "Tradicional", na Wikipédia e na Desciclopédia: percursos de sujeitos, saberes e línguas. In: Cristiane Dias (Org.) Formas de Mobilidade no Espaço E-Urbano: Sentido e Materialidade Digital. Série e-urbano. Vol. 2, 2013.

JOHNSON, T. Contrato de comunicação colaborativa na internet: um estudo de caso sobre a Wikipédia lusófona. Revista Mediação, Belo Horizonte, $\mathrm{n}^{\circ} 7,2^{\circ}$ semestre de 2008.

LIMA, V. W. Canais de interação na Wikipedia. Biblioteca online de Ciências da Comunicação, 2006.

MALINA, A.; AZEVEDO, Â. C. B. de. História da Perspectiva Sociocultural da Educação Física nos anos 1980 como Fundamento Crítico para a Educação Física Escolar. In: TELLES, S. de C. C.; TRIANI, F. Educação Física Escolar: múltiplos olhares. 1. Ed. Rio de Janeiro: Autobiografia, 2017, p. 28-50.

MALINA, A. Possibilidades e Limites ou o Máximo de Consciência Possível:

A Educação Física nos anos 1980. 191p. (Tese de doutorado). Programa de Pós Graduação em Educação Física da Universidade Gama Filho, 2005.

MONTEIRO, S. D. A relação das enciclopédias com os índices e a Web semântica: linhas de força para a organização e significação na pós-modernidade. Transinformação, Campinas , v. 29, n. 1, p. 15-25, Apr. 2017.

MOREIRA, W. Os colégios virtuais e a nova configuração da comunicação científicaCi. Inf., Brasília, v. 34, n. 1, p.57-63, jan./abr. 2005

PRIMO, A.F.T; RECUERO, R.C. Hipertexto cooperativo: uma análise da escrita coletiva a partir dos Blogs e da Wikipédia. Revista FAMECOS, Porto Alegre, $\mathrm{n}^{\mathrm{o}} 22$, dezembro, 2003.

RIBEIRO, A. L. R.; GOTTSCHALG-DUQUE, C. Wikipédia e Enciclopédia Britânica: informação confiável? Revista Brasileira de Biblioteconomia e Documentação. São Paulo, v.7, n.2, p. 172-185, jul./dez. 2011. 
RODRIGUES, C. V. Wikipédia: um monstro virtual. Uma análise sobre a interatividade e a autoria na construção cognitiva da enciclopédia virtual. Trabalho apresentado no XV Congresso de Ciências da Comunicação na Região Sudeste, Vitória, ES, 2010.

SILVEIRA, M. T; SILVA, A. R. A Semiótica do Futebol Televisivo: narrativas imersivas, intervalares e fragmentadas. Movimento, Porto Alegre, v. 22, n. 1, 211-222, jan./mar. de 2016.

TREVISOL, M.G; NOVELLO, J. D. Visibilidade midiática na pós-modernidade: reflexões acerca do papel da mídia na organização da estrutura social. Unoesc \& Ciência-ACHS, v. 5, n. 1, p. 131, 2014.

VIEIRA, M. V; CHRISTOFOLETTI, R. Confiabilidade no uso da wikipédia como fonte de pesquisa escolar.Revista Tecnologias na Educação. v.1, n.1, 2009. Disponível em: $\quad$ HTTP://tecnologiasnaeducacao.pro.br/revista/a1n1/art4.pdf Acesso em: 07/09/2018.

2008.

VIEIRA PINTO, Á. O Conceito de Tecnologia. Rio de Janeiro: Contraponto, 1 vol. 2005

WIKIMÉDIA. Mission. 2017. Acess in: 09/04/2017. Available from : https://meta.wikimedia.org/wiki/Mission/pt

WIKIPEDIA. Sobre a Wikipedia.2018. Acess in: 05/07/2018. Available from http://pt.wikipedia.org/wiki/Wikip\%C3\%A9dia:Sobre a Wikip\%C3\%A9dia> 